\title{
Randomized Global Transformation Approach for Dense Correspondence
}

Kihong Park

khpark7727@gmail.com

Seungryong Kim

srkim89@yonsei.ac.kr

Seungchul Ryu

ryus01@yonsei.ac.kr

Kwanghoon Sohn

khsohn@yonsei.ac.kr
School of Electrical and Electronic Engineering

Yonsei University

Seoul, Korea

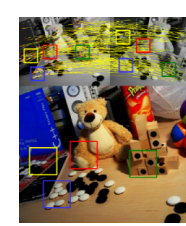

(a) Initial match

(b) $\mathbf{T}_{1}$

(c) $\mathbf{T}_{2}$

(d) $\mathbf{T}_{3}$

Figure 1: Intuitions of our approach. With sparse features in (a), there exist sparse correspondence sets for global transformation which provide reliable flow on each sub-region.

Motivation Recently, many researchers have begun to attempt to solve dense correspondence problem for more challenging images which have high variability in terms of photometric and/or geometric conditions [4].

For these challenging scenarios, there exist two principal bottlenecks which make conventional methods provide limited performances; (1) photometric variations derived from different camera specifications, illumination or exposure conditions, and (2) geometric variations derived from viewpoint changes, object pose changes, and non-rigid deformation for objects [1]. For the first bottleneck, many robust feature descriptors have been proposed to alleviate photometric variations. As a pioneering work, the SIFT flow [2] has shown satisfactory results on different image pairs having semantically similar property by employing the SIFT descriptor. However, geometric variations for the second bottleneck still remain unsolved due to its large search space, including translation (or flow), rotation, and scale, which induces extremely large computational complexity.

In this paper, we propose a randomized global transformation approach to estimate reliable correspondence between challenging image pairs having photometric and geometric deformation. Our approach starts from an intuition that geometric variations between two images can be formulated as piecewise transformation model as shown in Fig. 1. Finding the optimal global transformation for each pixel enables us to infer correspondence fields without computing geometric fields.

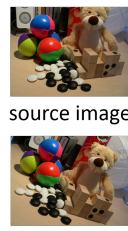

target image
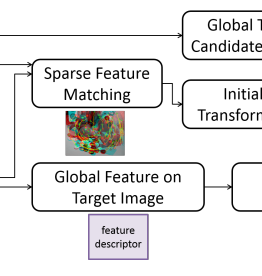
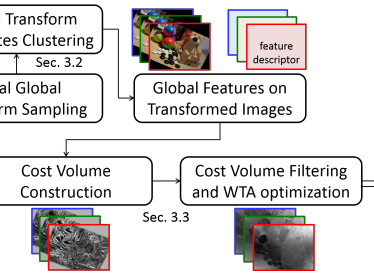

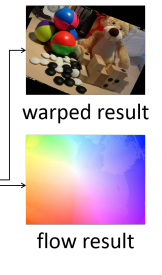

Figure 2: Framework of our randomized global transformation approach. It estimates initial global transformation candidates from sparse feature correspondences. The cost volume between global feature descriptors are then constructed. To infer reliable correspondence, it employs a cost volume filtering using fast edge-aware filtering.

Approach Fig. 2 shows overall framework of our approach. Finding the optimal global transformation for each pixel enables us to infer correspondence fields without computing geometric fields. To build reliable candidates of correspondence fields, we choose a flow hypothesis by leveraging a randomized sampling scheme from initial sparse matching fields. In order to reduce repeated transformation and provide robustness to initial outliers, we employ a clustering scheme on initial global transformation candidates. By using these global transformation candidates, dense descriptor is built on target image and transformed source images, which will be contributed to compute a cost volume between two image pairs. Furthermore, with edge-aware filtering scheme, we employ a cost filter scheme to provide a geometric smoothness.
Contributions The contribution of this paper can be summarized as follows. First of all, unlike conventional approaches that consider large search space extensively, our approach reduces search space labels by leveraging optimal global transformation, which provides accurate correspondence results while producing very low complexity. Secondly, in order to find reliable correspondences, we employ a cost filter scheme by leveraging fast edge-aware filtering. Thirdly, our approach is very simple but provides satisfactory results, and it further can be combined with any other feature descriptors and filtering approaches. Finally, we provide an intensive comparative study with state-of-the-art methods using various datasets.

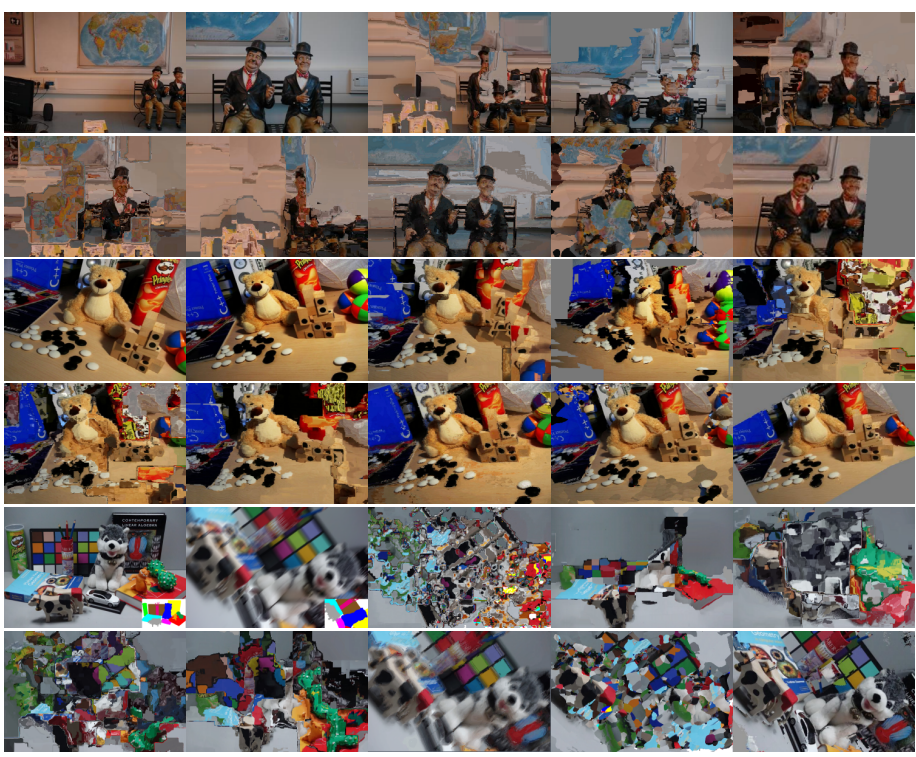

Figure 3: Comparison of dense correspondence for challenging non-rigid image pairs. (from top to bottom, from left to right) Source and target images, warping results from SIFT flow, SSF, SID, SegSID, SegSF, GPM, DFF, and proposed method.

Results Fig. 3 presents image warping results from estimated flow fields by computing dense correspondences from source to target images. These image pairs are non-rigid because their relationships cannot be expressed by just one global transformation [3]. We collected these image pairs among the geometric distorted images which were used in many previous researches, and thus can explain robustness property of our algorithm against various geometric challenging situation. Compared to conventional methods, our approach estimated reliably correspondence for challenging image pairs since even for challenging pairs the correspondence field can be modeled by piecewise parametric transformation models.

[1] J. Hur, H. Lim, C. Park, and S. C. Ahn. Generalized deformable spatial pyramid: Geometry-preserving dense correspondence estimation. In Proc. of CVPR, 2015.

[2] C. Liu, J. Yuen, and A. Torralba. Sift flow: Dense correspondence across scenes and its applications. IEEE Trans. PAMI, 33(5):978994, 2011.

[3] P. Weinzaepfel, J. Revaud, Z. Harchaoui, and C. Schmid. Deepflow: Large displacement optical flow with deep matching. In Proc. of ICCV, 2013.

[4] H. Yang, W. Lin, and J. Lu. Daisy filter flow: A generalized discrete approach to dense correspondences. In Proc. of CVPR, 2014. 